\title{
Existence results for a kind of fourth-order impulsive integral boundary value problems
}

Meng Sun and Yepeng Xing*

\section{${ }^{*}$ Correspondence:}

ypxingmath@gmail.com

Department of Mathematics,

Shanghai Normal University,

Shanghai, 200234, P.R. China

\begin{abstract}
In this paper we investigate the existence of solutions to a kind of fourth-order impulsive differential equations with integral boundary value conditions. By employing the Schauder fixed point theorem, we obtain sufficient conditions which ensure the system has at lease one solution. Also by using the contraction mapping theorem we get the uniqueness result. Finally an example is given to illustrate the effectiveness of our results.
\end{abstract}

Keywords: fourth order; impulsive; integral boundary conditions

\section{Introduction}

Fourth-order boundary value problems have attached much attention from many authors; for example, see Sun and Wang [1], Yao [2], O'Regan [3], Yang [4], Zhang [5], Gupta [6], Agarwal [7], Bonanno and Bella [8], and Han and Xu [9]. In particular, we would like to mention some results as follows. In [10], Zhang and Liu studied the following fourth-order four-point boundary value problem:

$$
\left\{\begin{array}{l}
\left(\phi_{p}\left(x^{\prime \prime}(t)\right)\right)^{\prime \prime}=\omega(t) f(t, x(t)), \quad t \in[0,1], \\
x(0)=0, \quad x(1)=a x(\xi), \\
x^{\prime \prime}(0)=0, \quad x^{\prime \prime}(1)=b x^{\prime \prime}(\eta),
\end{array}\right.
$$

where $0<\xi, \eta<1,0 \leq a<b<1$. By using the upper and lower solutions method, fixed point theorems, and the properties of the Green's functions $G(t, s)$ and $H(t, s)$, the authors gave sufficient conditions for the existence of one positive solution.

Zhou and Zhang [11] employed a new existence theory to study the fourth-order $p$-Laplacian elasticity problems:

$$
\left\{\begin{array}{l}
\left(\phi_{m}\left(y^{\prime \prime}\right)\right)^{\prime \prime}=F\left(t, y, y^{\prime \prime}\right), \quad 0<t<1, \\
a y(0)-b y^{\prime}(0)=\int_{0}^{1} g(s) y(s) d s, \\
a y(1)+b y^{\prime}(1)=\int_{0}^{1} g(s) y(s) d s, \\
\phi_{m}\left(y^{\prime \prime}(0)\right)=\phi_{m}\left(y^{\prime \prime}(1)\right)=\int_{0}^{1} h(t) \phi_{m}\left(y^{\prime \prime}(t)\right) d t,
\end{array}\right.
$$

where $a, b>0, J=[0,1], \phi_{m}(s)$ is an $m$-Laplace operator, i.e. $\phi_{m}(s)=|s|^{m-2} s, m>1,\left(\phi_{m}\right)^{-1}=$ $\phi_{m^{*}}, \frac{1}{m}+\frac{1}{m^{*}}=1, F:[0,1] \times R \times R \rightarrow R$ is continuous. In their paper, a new technique for dealing with the bending term of the fourth-order $p$-Laplacian elasticity problems was

(c) 2016 Sun and Xing. This article is distributed under the terms of the Creative Commons Attribution 4.0 International License (http://creativecommons.org/licenses/by/4.0/), which permits unrestricted use, distribution, and reproduction in any medium, provided you give appropriate credit to the original author(s) and the source, provide a link to the Creative Commons license, and indicate if changes were made. 
introduced and several new and more general results were obtained for the existence of at least single, double, or triple positive solutions.

Feng [12] studied a fourth-order boundary value problem with impulses and integral boundary conditions,

$$
\left\{\begin{array}{l}
\left(\phi_{p}\left(y^{\prime \prime}(t)\right)\right)^{\prime \prime}=f(t, y(t)), \quad t \in[0,1], t \neq t_{k}, k=1,2, \ldots, n, \\
\left.\Delta y^{\prime}\right|_{t=t_{k}}=-I_{k}\left(y\left(t_{k}\right)\right), \quad k=1,2, \ldots, n, \\
y(0)=y(1)=\int_{0}^{1} g(s) y(s) d s \\
\phi_{p}\left(y^{\prime \prime}(0)\right)=\phi_{p}\left(y^{\prime \prime}(1)\right)=\int_{0}^{1} h(s) \phi_{p}\left(y^{\prime \prime}(s)\right) d s .
\end{array}\right.
$$

By using a suitably constructed cone and fixed point theory for cones, the existence of multiple positive solutions was established. Some papers considered the existence, multiplicity, and nonexistence of positive solutions for fourth-order impulsive differential equations with one-dimensional $m$-Laplacian; for example, see [13-17]. Most recently Feng and Qiu [18], studied a fourth-order impulse integral boundary value problem with onedimensional $m$-Laplacian and deviating arguments:

$$
\left\{\begin{array}{l}
\left(\phi_{m}\left(y^{\prime \prime}\right)\right)^{\prime \prime}=\lambda \omega(t) f(t, y(\alpha(t))), \quad t \in J, t \neq t_{k}, k=1,2, \ldots, n, \\
\left.\Delta y^{\prime}\right|_{t=t_{k}}=-\mu I_{k}\left(t_{k}, y\left(t_{k}\right)\right), \quad k=1,2, \ldots, n, \\
a y(0)-b y^{\prime}(0)=\int_{0}^{1} g(s) y(s) d s \\
a y(1)+b y^{\prime}(1)=\int_{0}^{1} g(s) y(s) d s \\
\phi_{m}\left(y^{\prime \prime}(0)\right)=\phi_{m}\left(y^{\prime \prime}(1)\right)=\int_{0}^{1} h(t) \phi_{m}\left(y^{\prime \prime}(t)\right) d t .
\end{array}\right.
$$

We see that in the above system the right-hand side function $f$ has nothing to do with the term $y^{\prime}$, the jumping function $I_{k}$ does not contain the term $y^{\prime}\left(t_{k}\right)$. What is more, there is no restriction on the impulses for state function, i.e. $\left.\Delta y\right|_{t=t_{k}}$ does not appear. Definitely for more extensive applications, we would better consider the following boundary value problem:

$$
\left\{\begin{array}{l}
\left(\phi_{m}\left(y^{\prime \prime}\right)\right)^{\prime \prime}=f\left(t, y, y^{\prime}\right), \quad t \in J, t \neq t_{k}, k=1,2, \ldots, m \\
\left.\Delta y\right|_{t=t_{k}}=I_{k}\left(y\left(t_{k}\right)\right), \quad k=1,2, \ldots, m, \\
\left.\Delta y^{\prime}\right|_{t=t_{k}}=\bar{I}_{k}\left(y\left(t_{k}\right), y^{\prime}\left(t_{k}\right)\right), \quad k=1,2, \ldots, m \\
a y(0)-b y^{\prime}(0)=\int_{0}^{1} g(s) y(s) d s \\
a y(1)+b y^{\prime}(1)=\int_{0}^{1} g(s) y(s) d s \\
\phi_{m}\left(y^{\prime \prime}(0)\right)=\phi_{m}\left(y^{\prime \prime}(1)\right)=\int_{0}^{1} h(t) \phi_{m}\left(y^{\prime \prime}(t)\right) d t
\end{array}\right.
$$

where $a, b>0, J=[0,1], \phi_{m}(s)$ is an $m$-Laplace operator, i.e. $\phi_{m}(s)=|s|^{m-2} s, m>1,\left(\phi_{m}\right)^{-1}=$ $\left.\phi_{m^{*}}, \frac{1}{m}+\frac{1}{m^{*}}=1,0=t_{0}<t_{1}<t_{2}<\cdots<t_{k}<\cdots<t_{m}<t_{m+1}=1, f \in C U \times R^{n} \times R^{n}, R^{n}\right], I_{k} \in$ $C\left[R^{n}, R^{n}\right], \bar{I}_{k} \in C\left[R^{n} \times R^{n}, R^{n}\right],\left.\Delta y\right|_{t=t_{k}}=y\left(t_{k}^{+}\right)-y\left(t_{k}^{-}\right)$, here $y\left(t_{k}^{+}\right)$and $y\left(t_{k}^{-}\right)$represent the right-hand limit and left-hand limit of $y(t)$ at $t=t_{k}$, respectively. $\left.\Delta y^{\prime}\right|_{t=t_{k}}$ has a similar meaning for $y^{\prime}(t)$. In addition, $f, g$, and $h$ satisfy the following conditions.

(H1) $\left.f \in C U \times R^{n} \times R^{n}, R^{n}\right],\left.\Delta y\right|_{t=t_{k}}=y\left(t_{k}^{+}\right)-y\left(t_{k}^{-}\right)$, where $y\left(t_{k}^{+}\right)$and $y\left(t_{k}^{-}\right)$represent the right-hand limit and left-hand limit of $y(t)$ at $t=t_{k}$, respectively;

(H2) $I_{k} \in C\left[R^{n}, R^{n}\right], \bar{I}_{k} \in C\left[R^{n} \times R^{n}, R^{n}\right]$;

(H3) $g, h \in L^{1}[0,1]$ are nonnegative and $\xi \in[0, a), v \in[0,1)$ where

$$
\xi=\int_{0}^{1} g(t) d t, \quad v=\int_{0}^{1} h(t) d t .
$$


The remainder of the paper is organized as below. In Section 2, we give the expression of the solution to BVP (1.2). For this purpose, we do some computation and estimation of the Green's function. In Section 3, we show the existence and uniqueness of solutions to BVP (1.2) by the Schauder fixed point theorem and contraction mapping theorem. Section 4 gives an example to illustrate our main result.

\section{Preliminaries and lemmas}

We shall reduce problem (1.1) to an integral equation. To this aim, first, by means of the transformation

$$
\phi_{m}\left(y^{\prime \prime}(t)\right)=-x(t)
$$

we convert problem (1.1) into

$$
\left\{\begin{array}{l}
x^{\prime \prime}(t)+f\left(t, y, y^{\prime}\right)=0, \quad t \in J, \\
x(0)=x(1)=\int_{0}^{1} h(t) x(t) d t
\end{array}\right.
$$

and

$$
\left\{\begin{array}{l}
y^{\prime \prime}(t)=-\phi_{m^{*}}(x(t)), \quad t \in J, t \neq t_{k}, \\
\left.\Delta y\right|_{t=t_{k}}=I_{k}\left(y\left(t_{k}\right)\right), \quad k=1,2, \ldots, m, \\
\left.\Delta y^{\prime}\right|_{t=t_{k}}=\bar{I}_{k}\left(y\left(t_{k}\right), y^{\prime}\left(t_{k}\right)\right), \quad k=1,2, \ldots, m, \\
a y(0)-b y^{\prime}(0)=\int_{0}^{1} g(s) y(s) d s, \\
a y(1)+b y^{\prime}(1)=\int_{0}^{1} g(s) y(s) d s .
\end{array}\right.
$$

Lemma 2.1 If (H1), (H2), and (H3) hold, then problem (2.1) has a unique solution $x(t)$, which is given by

$$
x(t)=\int_{0}^{1} H(t, s) f\left(s, y, y^{\prime}\right) d s,
$$

where

$$
\begin{aligned}
& H(t, s)=G(t, s)+\frac{1}{1-v} \int_{0}^{1} G(s, \tau) h(\tau) d \tau, \\
& G(t, s)= \begin{cases}t(1-s), & 0 \leq t \leq s \leq 1 \\
s(1-t), & 0 \leq s \leq t \leq 1\end{cases}
\end{aligned}
$$

Proof Integrating (2.1) from 0 to $t$ we get

$$
\begin{aligned}
& x^{\prime}(t)-x^{\prime}(0)=-\int_{0}^{t} f\left(t, y, y^{\prime}\right) d t, \\
& x^{\prime}(t)=x^{\prime}(0)-\int_{0}^{t} f\left(t, y, y^{\prime}\right) d t .
\end{aligned}
$$

Integrating it again, we have

$$
x(t)-x(0)=x^{\prime}(0) t-\int_{0}^{t}(t-s) f\left(s, y, y^{\prime}\right) d s,
$$




$$
x(t)=x(0)+x^{\prime}(0) t-\int_{0}^{t}(t-s) f\left(s, y, y^{\prime}\right) d s
$$

From (2.1) we know that $x(0)=x(1)=\int_{0}^{1} h(t) x(t) d t$. Letting $t=1$ we then obtain

$$
x(1)=x(0)+x^{\prime}(0)-\int_{0}^{1}(1-s) f\left(s, y, y^{\prime}\right) d s
$$

Hence

$$
x^{\prime}(0)=\int_{0}^{1}(1-s) f\left(s, y, y^{\prime}\right) d s .
$$

Thus we get

$$
x(t)=\int_{0}^{1} h(t) x(t) d t+t \int_{0}^{1}(1-s) f\left(s, y, y^{\prime}\right) d s-\int_{0}^{t}(t-s) f\left(s, y, y^{\prime}\right) d s .
$$

In order to get the expression of $x(t)$, different from [3], we multiply both sides of (2.5) with function $h(t)$ and then integrating it from 0 to 1 , we have

$$
\begin{aligned}
\int_{0}^{1} h(t) x(t) d t= & \int_{0}^{1} h(s) d s \int_{0}^{1} h(t) x(t) d t+\int_{0}^{1} t h(t) d t \int_{0}^{1}(1-s) f\left(s, y, y^{\prime}\right) d s \\
& -\int_{0}^{1} h(t) d t \int_{0}^{t}(t-s) f\left(s, y, y^{\prime}\right) d s
\end{aligned}
$$

and

$$
\begin{aligned}
(1-v) \int_{0}^{1} h(t) x(t) d t= & \int_{0}^{1} t h(t) d t \int_{0}^{1}(1-s) f\left(s, y, y^{\prime}\right) d s \\
& -\int_{0}^{1} h(t) d t \int_{0}^{t}(t-s) f\left(s, y, y^{\prime}\right) d s .
\end{aligned}
$$

Hence,

$$
\begin{aligned}
\int_{0}^{1} h(t) x(t) d t= & \frac{1}{1-v}\left(\int_{0}^{1} t h(t) d t \int_{0}^{1}(1-s) f\left(s, y, y^{\prime}\right) d s\right. \\
& \left.-\int_{0}^{1} h(t) d t \int_{0}^{t}(t-s) f\left(s, y, y^{\prime}\right) d s\right) .
\end{aligned}
$$

Finally, we obtain

$$
\begin{aligned}
x(t)= & \frac{1}{1-v}\left(\int_{0}^{1} t h(t) d t \int_{0}^{1}(1-s) f\left(s, y, y^{\prime}\right) d s-\int_{0}^{1} h(t) d t \int_{0}^{t}(t-s) f\left(s, y, y^{\prime}\right) d s\right) \\
& +t \int_{0}^{1}(1-s) f\left(s, y, y^{\prime}\right) d s-\int_{0}^{1}(1-s) f\left(s, y, y^{\prime}\right) d s \\
= & \frac{1}{1-v}\left(\int_{0}^{1} \int_{0}^{1} t h(t)(1-s) f\left(s, y, y^{\prime}\right) d t d s-\int_{0}^{1} \int_{0}^{t} h(t)(t-s) f\left(s, y, y^{\prime}\right) d s d t\right) \\
& +\int_{0}^{1} t(1-s) f\left(s, y, y^{\prime}\right) d s-\int_{0}^{1}(1-s) f\left(s, y, y^{\prime}\right) d s
\end{aligned}
$$




$$
\begin{aligned}
= & \int_{0}^{1} \frac{1}{1-v}\left(\int_{0}^{1} t(1-s) h(t) d t-\int_{0}^{t}(t-s) h(t) d t\right) f\left(s, y, y^{\prime}\right) d s \\
& +\int_{0}^{1} t(1-s) f\left(s, y, y^{\prime}\right) d s-\int_{0}^{1}(1-s) f\left(s, y, y^{\prime}\right) d s \\
= & \int_{0}^{1} \frac{1}{1-v}\left(\int_{0}^{1} t(1-s) h(t) d t-\int_{0}^{t}(t-s) h(t) d t\right) f\left(s, y, y^{\prime}\right) d s \\
& +\int_{0}^{t} s(1-t) f\left(s, y, y^{\prime}\right) d s-\int_{t}^{1} t(1-s) f\left(s, y, y^{\prime}\right) d s .
\end{aligned}
$$

Thus

$$
x(t)=\int_{0}^{1} H(t, s) f\left(s, y, y^{\prime}\right) d s,
$$

where

$$
\begin{aligned}
& H(t, s)=G(t, s)+\frac{1}{1-v} \int_{0}^{1} G(s, \tau) h(\tau) d \tau \\
& G(t, s)= \begin{cases}t(1-s), & 0 \leq t \leq s \leq 1 \\
s(1-t), & 0 \leq s \leq t \leq 1\end{cases}
\end{aligned}
$$

This completes the proof.

Let $e(t)=t(1-t)$. Then from (2.3) and (2.4) we can prove that $H(t, s)$ and $G(t, s)$ have the following properties.

Lemma 2.2 Let $G(t, s)$ and $H(t, s)$ be given as in Lemma 2.1. Assume that $(\mathrm{H} 3)$ holds. Then we have

$$
\begin{aligned}
& H(t, s)>0, \quad G(t, s)>0, \quad \forall t, s \in(0,1), \\
& H(t, s) \geq 0, \quad G(t, s) \geq 0, \quad \forall t, s \in J, \\
& e(t) e(s) \leq G(t, s) \leq G(t, t)=t(1-t)=e(t) \leq \bar{e}=\max _{t \in J} e(t)=\frac{1}{4}, \quad \forall t, s \in J, \\
& \rho e(s) \leq H(t, s) \leq \gamma s(1-s)=\gamma e(s) \leq \frac{1}{4} \gamma, \quad \forall t, s \in J,
\end{aligned}
$$

where

$$
\gamma=\frac{1}{1-v}, \quad \rho=\frac{\int_{0}^{1} e(\tau) h(\tau) d \tau}{1-v} .
$$

Lemma 2.3 If (H1), (H2), and (H3) hold, then problem (2.2) has a unique solution $y(t)$ expressed in the form

$$
\begin{aligned}
y(t)= & \int_{0}^{1} H_{1}(t, s) \phi_{m^{*}}(x(s)) d s-\sum_{k=1}^{m} H_{1}\left(t, t_{k}\right) \bar{I}_{k}\left(y\left(t_{k}\right), y^{\prime}\left(t_{k}\right)\right) \\
& -\sum_{k=1}^{m} H_{2}(t) I_{k}\left(y\left(t_{k}\right)\right),
\end{aligned}
$$


where

$$
\begin{aligned}
& H_{1}(t, s)=G_{1}(t, s)+\frac{1}{a-\xi} \int_{0}^{1} G_{1}(s, \tau) g(\tau) d \tau, \\
& G_{1}(t, s)=\frac{1}{d} \begin{cases}(b+a s)(b+a(1-t)), & 0 \leq s \leq t \leq 1, \\
(b+a t)(b+a(1-s)), & 0 \leq t \leq s \leq 1,\end{cases} \\
& H_{2}(t)=\frac{a t-a-b}{a+2 b}+\frac{1}{a-\xi} \int_{0}^{1} \frac{a t-a-b}{a+2 b} g(t) d t,
\end{aligned}
$$

and $d=a(a+2 b)$.

Proof First, we assume that $t \in I_{i}, I_{i}=\left(t_{i}, t_{i+1}\right)(i=0,1,2, \ldots, m)$. Integrating both sides of (2.2) from $t_{i}$ to $t_{i+1}^{-}$, we get

$$
\begin{aligned}
& y^{\prime}\left(t_{1}^{-}\right)-y^{\prime}(0)=-\int_{0}^{t_{1}} \phi_{m^{*}}(x(t)) d t, \\
& y^{\prime}\left(t_{2}^{-}\right)-y^{\prime}\left(t_{1}^{+}\right)=-\int_{t_{1}}^{t_{2}} \phi_{m^{*}}(x(t)) d t, \\
& \ldots, \\
& y^{\prime}(t)-y^{\prime}\left(t_{i}^{+}\right)=-\int_{t_{i}}^{t} \phi_{m^{*}}(x(t)) d t .
\end{aligned}
$$

Adding the above equations, we find

$$
\begin{aligned}
& y^{\prime}(t)-y^{\prime}(0)-\sum_{k: t_{k}<t}\left(y^{\prime}\left(t_{k}^{+}\right)-y^{\prime}\left(t_{k}^{-}\right)\right)=-\int_{0}^{t} \phi_{m^{*}}(x(t)) d t, \\
& y^{\prime}(t)=y^{\prime}(0)+\sum_{k: t_{k}<t}\left(y^{\prime}\left(t_{k}^{+}\right)-y^{\prime}\left(t_{k}^{-}\right)\right)-\int_{0}^{t} \phi_{m^{*}}(x(t)) d t, \\
& y^{\prime}(t)=y^{\prime}(0)+\sum_{k: t_{k}<t} \bar{I}_{k}\left(y\left(t_{k}\right), y^{\prime}\left(t_{k}\right)\right)-\int_{0}^{t} \phi_{m^{*}}(x(t)) d t .
\end{aligned}
$$

Similarly, we can get

$$
\begin{aligned}
y(t)= & y(0)+y^{\prime}(0) t-\int_{0}^{t}(t-s) \phi_{m^{*}}(x(t)) d s \\
& +\sum_{k: t_{k}<t}\left(t-t_{k}\right) \bar{I}_{k}\left(y\left(t_{k}\right), y^{\prime}\left(t_{k}\right)\right)+\sum_{k: t_{k}<t} I_{k}\left(y\left(t_{k}\right)\right) .
\end{aligned}
$$

Let $t=1$ in (2.10) and (2.11). We obtain

$$
\left\{\begin{aligned}
b y^{\prime}(1)= & b y^{\prime}(0)+b \sum_{k: t_{k}<t} \bar{I}_{k}\left(y\left(t_{k}\right), y^{\prime}\left(t_{k}\right)\right)-b \int_{0}^{t} \phi_{m^{*}}(x(t)) d t, \\
a y(1)= & a y(0)+a y^{\prime}(0)-a \int_{0}^{1}(1-s) \phi_{m^{*}}(x(s)) d s \\
& +a \sum_{k: t_{k}<t}\left(1-t_{k}\right) \bar{I}_{k}\left(y\left(t_{k}\right), y^{\prime}\left(t_{k}\right)\right)+a \sum_{k: t_{k}<t} I_{k}\left(y\left(t_{k}\right)\right) .
\end{aligned}\right.
$$


It is easy to get

$$
\begin{aligned}
a y(1)+b y^{\prime}(1)= & a y(0)+(a+b) y^{\prime}(0)-a \int_{0}^{1}(1-s) \phi_{m^{*}}(x(s)) d s \\
& +a \sum_{k: t_{k}<t}\left(1-t_{k}\right) \bar{I}_{k}\left(y\left(t_{k}\right), y^{\prime}\left(t_{k}\right)\right)+a \sum_{k: t_{k}<t} I_{k}\left(y\left(t_{k}\right)\right) \\
& +b \sum_{k: t_{k}<t} \bar{I}_{k}\left(y\left(t_{k}\right), y^{\prime}\left(t_{k}\right)\right)-b \int_{0}^{t} \phi_{m^{*}}(x(t)) d t \\
= & \int_{0}^{1} g(s) y(s) d s,
\end{aligned}
$$

which implies

$$
\begin{aligned}
a y(0)+(a+b) y^{\prime}(0)= & a \int_{0}^{1}(1-s) \phi_{m^{*}}(x(s)) d s \\
& -a \sum_{k: t_{k}<t}\left(1-t_{k}\right) \bar{I}_{k}\left(y\left(t_{k}\right), y^{\prime}\left(t_{k}\right)\right) \\
& -a \sum_{k: t_{k}<t} I_{k}\left(y\left(t_{k}\right)\right)-b \sum_{k: t_{k}<t} \bar{I}_{k}\left(y\left(t_{k}\right), y^{\prime}\left(t_{k}\right)\right) \\
& +b \int_{0}^{t} \phi_{m^{*}}(x(t)) d t+\int_{0}^{1} g(s) y(s) d s .
\end{aligned}
$$

Then we have the following equations:

$$
\left\{\begin{array}{l}
a y(0)+(a+b) y^{\prime}(0) \\
=a \int_{0}^{1}(1-s) \phi_{m^{*}}(x(s)) d s-a \sum_{k: t_{k}<t}\left(1-t_{k}\right) \bar{I}_{k}\left(y\left(t_{k}\right), y^{\prime}\left(t_{k}\right)\right) \\
\quad-a \sum_{k: t_{k}<t} I_{k}\left(y\left(t_{k}\right)\right)-b \sum_{k: t_{k}<t} \bar{I}_{k}\left(y\left(t_{k}\right), y^{\prime}\left(t_{k}\right)\right) \\
\quad-b \int_{0}^{t} \phi_{m^{*}}(x(t)) d t+\int_{0}^{1} g(s) y(s) d s \\
a y(0)-b y^{\prime}(0)=\int_{0}^{1} g(s) y(s) d s .
\end{array}\right.
$$

Obviously,

$$
\begin{aligned}
y^{\prime}(0)= & \frac{1}{a+2 b}\left[a \int_{0}^{1}(1-s) \phi_{m^{*}}(x(t)) d s-a \sum_{k: t_{k}<t}\left(1-t_{k}\right) \bar{I}_{k}\left(y\left(t_{k}\right), y^{\prime}\left(t_{k}\right)\right)\right. \\
& \left.-a \sum_{k: t_{k}<t} I_{k}\left(y\left(t_{k}\right)\right)-b \sum_{k: t_{k}<t} \bar{I}_{k}\left(y\left(t_{k}\right), y^{\prime}\left(t_{k}\right)\right)+b \int_{0}^{t} \phi_{m^{*}}(x(t)) d t\right], \\
y(0)= & \frac{b}{a(a+2 b)}\left[a \int_{0}^{1}(1-s) \phi_{m^{*}}(x(s)) d s-a \sum_{k: t_{k}<t}\left(1-t_{k}\right) \bar{I}_{k}\left(y\left(t_{k}\right), y^{\prime}\left(t_{k}\right)\right)\right. \\
& \left.-a \sum_{k: t_{k}<t} I_{k}\left(y\left(t_{k}\right)\right)-b \sum_{k: t_{k}<t} \bar{I}_{k}\left(y\left(t_{k}\right), y^{\prime}\left(t_{k}\right)\right)+b \int_{0}^{t} \phi_{m^{*}}(x(t)) d t\right] \\
& +\frac{1}{a} \int_{0}^{1} g(s) y(s) d s .
\end{aligned}
$$


Substituting (2.10), (2.11) in (2.9), we have

$$
\begin{aligned}
y(t)= & \frac{b}{a(a+2 b)}\left[a \int_{0}^{1}(1-s) \phi_{m^{*}}(x(s)) d s-a \sum_{k: t_{k}<t}\left(1-t_{k}\right) \bar{I}_{k}\left(y\left(t_{k}\right), y^{\prime}\left(t_{k}\right)\right)\right. \\
& \left.-a \sum_{k: t_{k}<t} I_{k}\left(y\left(t_{k}\right)\right)-b \sum_{k: t_{k}<t} \bar{I}_{k}\left(y\left(t_{k}\right), y^{\prime}\left(t_{k}\right)\right)+b \int_{0}^{t} \phi_{m^{*}}(x(t)) d t\right] \\
& +\frac{1}{a} \int_{0}^{1} g(s) y(s) d s+\frac{t}{a+2 b}\left[a \int_{0}^{1}(1-s) \phi_{m^{*}}(x(s)) d s\right. \\
& -a \sum_{k: t_{k}<t}\left(1-t_{k}\right) \bar{I}_{k}\left(y\left(t_{k}\right), y^{\prime}\left(t_{k}\right)\right)-a \sum_{k: t_{k}<t} I_{k}\left(y\left(t_{k}\right)\right) \\
& \left.-b \sum_{k: t_{k}<t} \bar{I}_{k}\left(y\left(t_{k}\right), y^{\prime}\left(t_{k}\right)\right)+b \int_{0}^{t} \phi_{m^{*}}(x(t)) d t\right] \\
& -\int_{0}^{t}(t-s) \phi_{m^{*}}(x(t)) d s+\sum_{k: t_{k}<t}\left(t-t_{k}\right) \bar{I}_{k}\left(y\left(t_{k}\right), y^{\prime}\left(t_{k}\right)\right)+\sum_{k: t_{k}<t} I_{k}\left(y\left(t_{k}\right)\right) .
\end{aligned}
$$

In a similar way as the proof of (2.6) in Lemma 2.1, we get

$$
\begin{aligned}
\int_{0}^{1} g(s) y(s) d s= & \frac{a}{a-\xi}\left\{\frac { b } { a ( a + 2 b ) } \left[\int_{0}^{1} g(t) d t a \int_{0}^{1}(1-s) \phi_{m^{*}}(x(t)) d s\right.\right. \\
& -a \sum_{k: t_{k}<t}\left(1-t_{k}\right) \bar{I}_{k}\left(y\left(t_{k}\right), y^{\prime}\left(t_{k}\right)\right)-a \sum_{k: t_{k}<t} I_{k}\left(y\left(t_{k}\right)\right) \\
& \left.-b \sum_{k: t_{k}<t} \bar{I}_{k}\left(y\left(t_{k}\right), y^{\prime}\left(t_{k}\right)\right)+b \int_{0}^{t} \phi_{m^{*}}(x(t)) d t\right] \\
& +\frac{t}{a+2 b} \int_{0}^{1} g(t) d t\left[a \int_{0}^{1}(1-s) \phi_{m^{*}}(x(s)) d s\right. \\
& -a \sum_{k: t_{k}<t}\left(1-t_{k}\right) \bar{I}_{k}\left(y\left(t_{k}\right), y^{\prime}\left(t_{k}\right)\right)-a \sum_{k: t_{k}<t} I_{k}\left(y\left(t_{k}\right)\right) \\
& \left.-b \sum_{k: t_{k}<t} \bar{I}_{k}\left(y\left(t_{k}\right), y^{\prime}\left(t_{k}\right)\right)+b \int_{0}^{t} \phi_{m^{*}}(x(t)) d t\right] \\
& -\int_{0}^{1} g(t) d t \int_{0}^{t}(t-s) \phi_{m^{*}}(x(s)) d s \\
& \left.+\sum_{k: t_{k}<t}\left(t-t_{k}\right) \bar{I}_{k}\left(y\left(t_{k}\right), y^{\prime}\left(t_{k}\right)\right) \int_{0}^{1} g(t) d t+\int_{0}^{1} g(t) \sum_{k: t_{k}<t} I_{k}\left(y\left(t_{k}\right)\right) d t\right\} .
\end{aligned}
$$

Hence, we finally get

$$
\begin{aligned}
y(t)= & \frac{b}{a(a+2 b)}\left[a \int_{0}^{1}(1-s) \phi_{m^{*}}(x(s)) d s-a \sum_{k: t_{k}<t}\left(1-t_{k}\right) \bar{I}_{k}\left(y\left(t_{k}\right), y^{\prime}\left(t_{k}\right)\right)\right. \\
& \left.-a \sum_{k: t_{k}<t} I_{k}\left(y\left(t_{k}\right)\right)-b \sum_{k: t_{k}<t} \bar{I}_{k}\left(y\left(t_{k}\right), y^{\prime}\left(t_{k}\right)\right)+b \int_{0}^{t} \phi_{m^{*}}(x(t)) d t\right]
\end{aligned}
$$




$$
\begin{aligned}
& +\frac{a}{a-\xi}\left\{\frac { b } { a ( a + 2 b ) } \left[\int_{0}^{1} g(t) d t a \int_{0}^{1}(1-s) \phi_{m^{*}}(x(s)) d s\right.\right. \\
& -a \sum_{k: t_{k}<t}\left(1-t_{k}\right) \bar{I}_{k}\left(y\left(t_{k}\right), y^{\prime}\left(t_{k}\right)\right)-a \sum_{k: t_{k}<t} I_{k}\left(y\left(t_{k}\right)\right) \\
& \left.-b \sum_{k: t_{k}<t} \bar{I}_{k}\left(y\left(t_{k}\right), y^{\prime}\left(t_{k}\right)\right)+b \int_{0}^{t} \phi_{m^{*}}(x(t)) d t\right] \\
& +\frac{t}{a+2 b} \int_{0}^{1} g(t) d t\left[a \int_{0}^{1}(1-s) \phi_{m^{*}}(x(s)) d s-a \sum_{k: t_{k}<t}\left(1-t_{k}\right) \bar{I}_{k}\left(y\left(t_{k}\right), y^{\prime}\left(t_{k}\right)\right)\right. \\
& \left.-a \sum_{k: t_{k}<t} I_{k}\left(y\left(t_{k}\right)\right)-b \sum_{k: t_{k}<t} \bar{I}_{k}\left(y\left(t_{k}\right), y^{\prime}\left(t_{k}\right)\right)+b \int_{0}^{t} \phi_{m^{*}}(x(t)) d t\right] \\
& -\int_{0}^{1} g(t) d t \int_{0}^{t}(t-s) \phi_{m^{*}}(x(s)) d s+\sum_{k: t_{k}<t}\left(t-t_{k}\right) \bar{I}_{k}\left(y\left(t_{k}\right), y^{\prime}\left(t_{k}\right)\right) \int_{0}^{1} g(t) d t \\
& \left.+\int_{0}^{1} g(t) d t \sum_{k: t_{k}<t} I_{k}\left(y\left(t_{k}\right)\right)\right\} \\
& +\frac{t}{a+2 b}\left[a \int_{0}^{1}(1-s) \phi_{m^{*}}(x(s)) d s-a \sum_{k: t_{k}<t}\left(1-t_{k}\right) \bar{I}_{k}\left(y\left(t_{k}\right), y^{\prime}\left(t_{k}\right)\right)\right. \\
& \left.-a \sum_{k: t_{k}<t} I_{k}\left(y\left(t_{k}\right)\right)-b \sum_{k: t_{k}<t} \bar{I}_{k}\left(y\left(t_{k}\right), y^{\prime}\left(t_{k}\right)\right)+b \int_{0}^{t} \phi_{m^{*}}(x(t)) d t\right] \\
& -\int_{0}^{t}(t-s) \phi_{m^{*}}(x(s)) d s+\sum_{k: t_{k}<t}\left(t-t_{k}\right) \bar{I}_{k}\left(y\left(t_{k}\right), y^{\prime}\left(t_{k}\right)\right)+\sum_{k: t_{k}<t} I_{k}\left(y\left(t_{k}\right)\right) \text {. }
\end{aligned}
$$

Hence

$$
y(t)=\int_{0}^{1} H_{1}(t, s) \phi_{m^{*}}(x(s)) d s-\sum_{k=1}^{m} H_{1}\left(t, t_{k}\right) \bar{I}_{k}\left(y\left(t_{k}\right), y^{\prime}\left(t_{k}\right)\right)-\sum_{k=1}^{m} H_{2}(t) I_{k}\left(y\left(t_{k}\right)\right),
$$

where

$$
\begin{aligned}
& H_{1}(t, s)=G_{1}(t, s)+\frac{1}{a-\xi} \int_{0}^{1} G_{1}(s, \tau) g(\tau) d \tau, \\
& G_{1}(t, s)=\frac{1}{d}\left\{\begin{array}{l}
(b+a s)(b+a(1-t)), \quad 0 \leq s \leq t \leq 1, \\
(b+a t)(b+a(1-s)), \quad 0 \leq t \leq s \leq 1,
\end{array}\right. \\
& H_{2}(t)=\frac{a t-a-b}{a+2 b}+\frac{1}{a-\xi} \int_{0}^{1} \frac{a t-a-b}{a+2 b} g(t) d t
\end{aligned}
$$

Then the proof is completed.

It follows from (2.7), (2.8), and (2.9) that $H_{1}(t, s), G_{1}(t, s)$, and $H_{2}(t)$ have the following properties.

Lemma 2.4 Suppose (H3) holds and assume that $G_{1}(t, s)$ and $H_{1}(t, s)$ are given as in Lemma 2.3. Then we have

$$
\frac{1}{d} b^{2} \leq G_{1}(t, s) \leq G_{1}(s, s) \leq \frac{(a+b)^{2}}{d}, \quad \forall t, s \in J,
$$




$$
\begin{aligned}
& \rho_{1} \leq H_{1}(t, s) \leq \frac{a}{a-\xi} G_{1}(s, s) \leq \rho_{2}, \quad \forall t, s \in J \\
& H_{2}(t) \leq \rho_{2},
\end{aligned}
$$

where

$$
\delta=\frac{b}{a+b}, \quad \rho_{1}=\frac{b^{2} \gamma^{1}}{a+2 b}, \quad \rho_{2}=\frac{a(a+b)^{2}}{(a-\xi) d} .
$$

Proof Clearly, it follows from the definition of $G_{1}(t, s)$ that (2.12) holds. Now we show that (2.13) and (2.14) are true.

In fact, for $t \in[\zeta, 1]$ and $s \in J$, we have

$$
\begin{aligned}
H_{1}(t, s) & =G_{1}(t, s)+\frac{1}{a-\xi} \int_{0}^{1} G_{1}(s, \tau) g(\tau) d \tau \\
& \leq G_{1}(s, s)+\frac{\xi}{a-\xi} G_{1}(s, s) \leq \frac{a(a+b)^{2}}{(a-\xi) d}=\rho_{2}, \\
H_{2}(t)= & \frac{a t-a-b}{a+2 b}+\frac{1}{a-\xi} \int_{0}^{1} \frac{a t-a-b}{a+2 b} g(t) d t \leq \frac{a t}{a+2 b}+\frac{\xi}{a-\xi} \frac{a t}{a+2 b}, \\
\frac{a t}{a+2 b} & \leq \frac{a}{a+2 b} \leq \frac{(a+b)^{2}}{a+2 b} .
\end{aligned}
$$

Consequently,

$$
H_{2}(t) \leq \frac{(a+b)^{2}}{a+2 b}+\frac{\xi}{a-\xi} \frac{(a+b)^{2}}{a+2 b}=\frac{a(a+b)^{2}}{(a-\xi) d}=\rho_{2} .
$$

This completes the proof.

Combining Lemma 2.1 with Lemma 2.3, we can get directly the following result.

Lemma 2.5 Assume that (H1)-(H3) hold. Then $y(t)$ has the following form:

$$
\begin{aligned}
y(t)= & \int_{0}^{1} H_{1}(t, s) \phi_{m^{*}}\left(\int_{0}^{1} H(s, \tau) f\left(\tau, y(\tau), y^{\prime}(\tau)\right) d \tau\right) d s \\
& -\sum_{k=1}^{m} H_{1}\left(t, t_{k}\right) \bar{I}_{k}\left(y\left(t_{k}\right), y^{\prime}\left(t_{k}\right)\right)-\sum_{k=1}^{m} H_{2}(t) I_{k}\left(y\left(t_{k}\right)\right) .
\end{aligned}
$$

Proof The conclusion is so straightforward that we omit it here.

We next give some notations and a fixed point theorem which will be used to prove our main results. Let

$$
\begin{aligned}
P C^{1}\left[J, R^{n}\right]= & \left\{y: J \rightarrow R^{n} \mid y^{\prime}(t) \text { is continuous when } t \neq t_{k}, y\left(t_{k}^{+}\right), y\left(t_{-}^{+}\right), y^{\prime}\left(t_{k}^{+}\right), y^{\prime}\left(t_{k}^{-}\right)\right. \\
& \text {exist and } \left.y\left(t_{k}^{-}\right)=y\left(t_{k}\right), k=1,2, \ldots, m\right\} .
\end{aligned}
$$

Clearly, $P C^{1}\left[J, R^{n}\right]$ is a Banach space with the norm $\|y\|_{P C^{1}}=\max \left\{\|y\|_{P C^{1}},\left\|y^{\prime}\right\|_{P C^{1}}\right\}$. 
Lemma $2.6[19] H \subset P C^{1}\left[J, R^{n}\right]$ is a relatively compact set if and only if $\forall y \in H, y$ and $y^{\prime}$ are uniformly bounded in $J$ and equi-continuous on $J_{k}(k=0,1,2, \ldots, m)$.

Definition 2.1 A function $y \in P C^{1}$ is said to be a solution of (1.1) if it satisfies every equation in system (1.1).

Lemma 2.7 (Schauder fixed point theorem) If $K$ is a nonempty convex subset of a Banach space $V$ and $T$ is a continuous mapping of $K$ into itself such that $T(K)$ is contained in a compact subset of $K$, then $T$ has a fixed point.

Definition 2.2 Define an operator $A: P C^{1}\left[U, R^{n}\right] \rightarrow P C^{1}\left[J, R^{n}\right]$ by

$$
\begin{aligned}
(A y)(t)= & \int_{0}^{1} H_{1}(t, s) \phi_{m^{*}}\left(\int_{0}^{1} H(s, \alpha) f\left(\alpha, y, y^{\prime}\right) d \alpha\right) d s \\
& -\sum_{k=1}^{m} H_{1}\left(t, t_{k}\right) \bar{I}_{k}\left(y\left(t_{k}\right), y^{\prime}\left(t_{k}\right)\right)-\sum_{k=1}^{m} H_{2}(t) I_{k}\left(y\left(t_{k}\right)\right) .
\end{aligned}
$$

Lemma 2.8 Assume that (H1)-(H3) hold. Then $y(t) \in J$ is a fixed point of $A$ if and only if $y(t)$ is a solution of problem (1.1).

Lemma 2.9 The operator $A: P C^{1}\left[J, R^{n}\right] \rightarrow P C^{1}\left[J, R^{n}\right]$ is completely continuous.

Proof According to (2.16) we have

$$
\begin{aligned}
(A y)^{\prime}(t)= & \int_{0}^{1} H_{1 t}^{\prime}(t, s) \phi_{m^{*}}\left(\int_{0}^{1} H(s, \tau) f\left(\tau, y, y^{\prime}\right) d \tau\right) d s \\
& -\sum_{k=1}^{m} H_{1}^{\prime}\left(t, t_{k}\right) \bar{I}_{k}\left(y\left(t_{k}\right), y^{\prime}\left(t_{k}\right)\right)-\sum_{k=1}^{m} H_{2}^{\prime}(t) I_{k}\left(y\left(t_{k}\right)\right) .
\end{aligned}
$$

From (2.16) and (2.17) we know that $A: P C^{1}\left[J, R^{n}\right] \rightarrow P C^{1}\left[J, R^{n}\right]$ is continuous. For any bounded set $S \in P C^{1}\left[J, R^{n}\right]$, and any function $y(t) \in S$, we see that $(A y)(t)$ and $(A y)^{\prime}(t)$ are uniformly bounded and equi-continuous on $J_{k}(k=0,1,2, \ldots, m)$. Hence, according to Lemma 2.6 we see that $A(S)$ is a relatively compact set, therefore $A$ is a completely continuous operator.

\section{Main results}

Let

$$
\begin{aligned}
& \beta=\varlimsup_{\|y\|+\left\|y^{\prime}\right\| \rightarrow \infty}\left(\frac{\left\|f\left(t, y, y^{\prime}\right)\right\|}{\phi_{m^{*}}\left(\|y\|+\left\|y^{\prime}\right\|\right)}\right), \\
& \beta_{k}=\varlimsup_{\|y\| \rightarrow \infty}\left(\frac{\left\|I_{k}(y)\right\|}{\|y\|}\right), \\
& \bar{\beta}_{k}=\varlimsup_{\|y\|+\left\|y^{\prime}\right\| \rightarrow \infty}\left(\frac{\left\|\bar{I}_{k}\left(y, y^{\prime}\right)\right\|}{\|y\|+\left\|y^{\prime}\right\|}\right) \quad(k=1,2, \ldots, m) .
\end{aligned}
$$


Theorem 3.1 Assume that (H1)-(H3) hold. Let $\eta=\max \left\{\eta_{1}, \eta_{2}\right\}<1$, then (1.1) has at least one solution, where

$$
\begin{aligned}
& \eta_{1}=2 \rho_{2} \phi_{m^{*}}\left(\frac{\gamma \beta}{4}\right)+2 m \rho_{2} \bar{\beta}_{k}+m \rho_{2} \beta_{k}, \\
& \eta_{2}=2 \rho_{3} \phi_{m^{*}}\left(\frac{\gamma \beta}{4}\right)+2 m \rho_{3} \bar{\beta}_{k}+m \rho_{2} \beta_{k} .
\end{aligned}
$$

Proof From the definition of $\beta$, there exists $N>0$, s.t.

$$
\left\|f\left(t, y, y^{\prime}\right)\right\| \leq \beta \phi_{m^{*}}\left(\|y\|+\left\|y^{\prime}\right\|\right), \quad \forall t \in J, \phi_{m^{*}}\left(\|y\|+\left\|y^{\prime}\right\|\right) \geq N .
$$

Similarly, we get

$$
\begin{aligned}
& \left\|I_{k}(y)\right\| \leq \beta_{k}\|y\|, \\
& \left\|\bar{I}_{k}\left(y, y^{\prime}\right)\right\| \leq \bar{\beta}_{k}\left(\|y\|+\left\|y^{\prime}\right\|\right), \quad \forall y, y^{\prime} \in R^{n}(k=1,2, \ldots, m) .
\end{aligned}
$$

This together with (2.14), (2.15) implies that

$$
\begin{aligned}
\|(A y)(t)\|= & \| \int_{0}^{1} H_{1}(t, s) \phi_{m^{*}}\left(\int_{0}^{1} H(s, \tau) f\left(\tau, y, y^{\prime}\right) d \tau\right) d s \\
& -\sum_{k=1}^{m} H_{1}\left(t, t_{k}\right) \bar{I}_{k}\left(y\left(t_{k}\right), y^{\prime}\left(t_{k}\right)\right)-\sum_{k=1}^{m} H_{2}(t) I_{k}\left(y\left(t_{k}\right)\right) \| \\
\leq & \left\|\rho_{2} \phi_{m^{*}}\left(\int_{0}^{1} H(s, \tau) f\left(\tau, y(\tau), y^{\prime}(\tau)\right) d \tau\right)\right\| \\
& +m \rho_{2}\left(\bar{\beta}_{k}\left(\|y\|+\left\|y^{\prime}\right\|\right)\right)+m \rho_{2} \beta_{k}\|y\| \\
\leq & \rho_{2} \phi_{m^{*}}\left(\frac{\gamma}{4} \beta \phi_{m}\left(\|y\|+\left\|y^{\prime}\right\|\right)\right)+2 m \rho_{2} \bar{\beta}_{k}\|y\|+m \rho_{2} \beta_{k}\|y\| \\
\leq & 2 \rho_{2} \phi_{m^{*}}\left(\frac{\gamma \beta}{4}\right)\|y\|+2 m \rho_{2} \bar{\beta}_{k}\|y\|+m \rho_{2} \beta_{k}\|y\| \\
\leq & \left(2 \rho_{2} \phi_{m^{*}}\left(\frac{\gamma \beta}{4}\right)+2 m \rho_{2} \bar{\beta}_{k}+m \rho_{2} \beta_{k}\right)\|y\| \\
\leq & \eta_{1}\|y\| \\
\leq & \|y\|
\end{aligned}
$$

where $\eta_{1}=2 \rho_{2} \phi_{m^{*}}\left(\frac{\gamma \beta}{4}\right)+2 m \rho_{2} \bar{\beta}_{k}+m \rho_{2} \beta_{k}$.

From (2.7), (2.8), and (2.9), we have

$$
\begin{aligned}
& H_{1 t}^{\prime}(t, s)=G_{1 t}^{\prime}(t, s)=\frac{1}{d} \begin{cases}-a(b+a s), & 0 \leq s \leq t \leq 1, \\
a(b+a(1-s)), & 0 \leq t \leq s \leq 1,\end{cases} \\
& \max _{t, s \in J, t \neq s}\left|H_{1 t}^{\prime}(t, s)\right|=\max _{t, s \in J, t \neq s}\left|G_{1 t}^{\prime}(t, s)\right| \leq \frac{1}{d} a(a+b)=\frac{a+b}{a+2 b}=\rho_{3},
\end{aligned}
$$


and

$$
H_{2}^{\prime}(t)=\frac{a}{a+2 b} \leq \rho_{2}
$$

which together with (2.17) imply

$$
\begin{aligned}
\left\|(A y)^{\prime}(t)\right\|= & \| \int_{0}^{1} H_{1 t}^{\prime}(t, s) \phi_{m^{*}}\left(\int_{0}^{1} H(s, \tau) f\left(\tau, y, y^{\prime}\right) d \tau\right) d s \\
& -\sum_{k=1}^{m} H_{1}^{\prime}\left(t, t_{k}\right) \bar{I}_{k}\left(y\left(t_{k}\right), y^{\prime}\left(t_{k}\right)\right)-\sum_{k=1}^{m} H_{2}^{\prime}(t) I_{k}\left(y\left(t_{k}\right)\right) \| \\
\leq & \rho_{3} \phi_{m^{*}}\left(\frac{\gamma}{4} \beta \phi_{m}\left(\|y\|+\left\|y^{\prime}\right\|\right)\right)+m \rho_{2}\left(\bar{\beta}_{k}\left(\|y\|+\left\|y^{\prime}\right\|\right)\right)+m \rho_{2} \beta_{k}\|y\| \\
\leq & \rho_{3} \phi_{m^{*}}\left(\frac{\gamma \beta}{4}\right)\left(\|y\|+\left\|y^{\prime}\right\|\right)+2 m \rho_{3} \bar{\beta}_{k}\|y\|+m \rho_{2} \beta_{k}\|y\| \\
\leq & \left(2 \rho_{3} \phi_{m^{*}}\left(\frac{\gamma \beta}{4}\right)+2 m \rho_{3} \bar{\beta}_{k}+m \rho_{2} \beta_{k}\right)\|y\| \\
\leq & \eta_{2}\|y\| \\
\leq & \|y\|,
\end{aligned}
$$

where $\eta_{2}=2 \rho_{3} \phi_{m^{*}}\left(\frac{\gamma \beta}{4}\right)+2 m \rho_{3} \bar{\beta}_{k}+m \rho_{2} \beta_{k}$.

On the other hand, according to Lemma 2.9, we know operator $A$ is a completely continuous operator. Together with Lemma 2.6 (Schauder fixed point theorem), we know $A$ has a fixed point in $P C^{1}\left[J, R^{n}\right]$.

Theorem 3.2 Assume that (H1)-(H3) hold. If there exist nonnegative real numbers $\alpha, \alpha_{k}$, $\bar{\alpha}_{k}$, s.t.

$$
\begin{aligned}
& \left\|\phi_{m^{*}}(x)-\phi_{m^{*}}(y)\right\| \leq \alpha(\|x-y\|), \\
& \left\|\bar{I}_{k}\left(x, x^{\prime}\right)-\bar{I}_{k}\left(y, y^{\prime}\right)\right\| \leq \bar{\alpha}_{k}\left(\|x-y\|+\left\|x^{\prime}-y^{\prime}\right\|\right), \\
& \left\|I_{k}(x)-I_{k}(y)\right\| \leq \alpha_{k}\|x-y\|,
\end{aligned}
$$

and $\xi=\max \left\{\xi_{1}, \xi_{2}\right\}<1$, then (1.1) has a unique solution, where

$$
\begin{aligned}
& \xi_{1}=\rho_{2}\left(2 \alpha+2 m \bar{\alpha}_{k}+m \alpha_{k}\right), \\
& \xi_{2}=2 \rho_{3} \alpha+2 m \rho_{3} \bar{\alpha}_{k}+m \rho_{2} .
\end{aligned}
$$

Proof From (2.16) and (2.17), similar to the proof of Theorem 3.1, we get

$$
\begin{aligned}
(A x)(t)-(A y)(t)= & \int_{0}^{1} H_{1}(t, s)\left(\phi_{m^{*}}(x)-\phi_{m^{*}}(y)\right)+\sum_{k=1}^{m} H_{1}\left(t, t_{k}\right)\left(\bar{I}_{k}\left(y, y^{\prime}\right)-\bar{I}_{k}\left(x, x^{\prime}\right)\right) \\
& +\sum_{k=1}^{m} H_{2}(t)\left(I_{k}(y)-I_{k}(x)\right) .
\end{aligned}
$$


Computing straightforwardly we have

$$
\begin{aligned}
\|(A x)(t)-(A y)(t)\| \leq & \rho_{2} \alpha(\|x-y\|)+\sum_{k=1}^{m} \rho_{2} \bar{\alpha}_{k}\left(\|x-y\|+\left\|x^{\prime}-y^{\prime}\right\|\right) \\
& +\sum_{k=1}^{m} \rho_{2} \alpha_{k}(\|x-y\|) \\
\leq & \rho_{2}\left(\alpha+2 m \bar{\alpha}_{k}+m \alpha_{k}\right)\|x-y\|_{P C^{1}} \\
= & \xi 1\|x-y\|_{P C^{1}} \\
\leq & \xi\|x-y\|_{P C^{1}} .
\end{aligned}
$$

Also we obtain

$$
\begin{aligned}
(A x)^{\prime}(t)-(A y)^{\prime}(t)= & \int_{0}^{1} H_{1 t}^{\prime}(t, s)\left(\phi_{m^{*}}(x)-\phi_{m^{*}}(y)\right) \\
& +\sum_{k=1}^{m} H_{1}^{\prime}\left(t, t_{k}\right)\left(\bar{I}_{k}\left(y, y^{\prime}\right)-\bar{I}_{k}\left(x, x^{\prime}\right)\right) \\
& +\sum_{k=1}^{m} H_{2}^{\prime}(t)\left(I_{k}(y)-I_{k}(x)\right), \\
\left\|(A x)^{\prime}(t)-(A y)^{\prime}(t)\right\| & \leq \rho_{3} \alpha\|x-y\|+m \rho_{3} \bar{\alpha}_{k}\left(\|x-y\|+\left\|x^{\prime}-y^{\prime}\right\|\right)+m \rho_{2} \alpha_{k}\|x-y\| \\
& \leq\left(\rho_{3} \alpha+2 m \rho_{3} \bar{\alpha}_{k}+m \rho_{2} \alpha_{k}\right)\|x-y\|_{P C^{1}} \\
& =\xi_{2}\|x-y\|_{P C^{1}} \\
& \leq \xi\|x-y\|_{P C^{1}} .
\end{aligned}
$$

It follows from $\xi<1$ that $A$ has a unique fixed point and therefore (1.1) has a unique solution.

\section{Example}

In this section, we will illustrate the main results by a simple example.

Let $n=1, t_{1}=\frac{1}{2}, a=b=1, I_{1}\left(y\left(t_{1}\right)\right)=\bar{I}_{1}\left(y\left(t_{1}, y^{\prime}\left(t_{1}\right)\right)\right)=\frac{1}{2}, f\left(t, y, y^{\prime}\right)=\sqrt[3]{t-y+y^{\prime}}-\frac{1}{42} y^{\prime}-$ $3 \ln \left(1+y^{2}\right)$, and $g(s)=\frac{1}{3}, h(t)=\frac{1}{2}, m=3$ in $\phi_{m}$. Then equation (1.1) turns to the following equation:

$$
\left\{\begin{array}{l}
\left(\phi_{3}\left(y^{\prime \prime}\right)\right)^{\prime \prime}=\sqrt[3]{t-y+y^{\prime}}-\frac{1}{41} y^{\prime}-3 \ln \left(1+y^{2}\right), \quad t \in J, t \neq \frac{1}{2} \\
\left.\Delta y\right|_{t=\frac{1}{2}}=\frac{1}{2} \\
\left.\Delta y^{\prime}\right|_{t=\frac{1}{2}}=\frac{1}{2} \\
y(0)-y^{\prime}(0)=\int_{0}^{1} \frac{1}{3} y(s) d s \\
y(1)+y^{\prime}(1)=\int_{0}^{1} \frac{1}{3} y(s) d s \\
\phi_{3}\left(y^{\prime \prime}(0)\right)=\phi_{3}\left(y^{\prime \prime}(1)\right)=\int_{0}^{1} \frac{1}{2} \phi_{3}\left(y^{\prime \prime}(t)\right) d t
\end{array}\right.
$$

Following Theorem 3.1, we have the following result.

Theorem 4.1 The problem (4.1) has at least one positive solution. 
Proof Obviously, $\left.f\left(t, y, y^{\prime}\right) \in C U \times R^{n} \times R^{n}, R^{n}\right], I_{1}\left(y\left(t_{1}\right)\right) \in C\left[R^{n}, R^{n}\right], \bar{I}_{1}\left(y\left(t_{1}, y^{\prime}\left(t_{1}\right)\right)\right) \in$ $C\left[R^{n} \times R^{n}, R^{n}\right]$. From (4.1), we get

$$
\begin{aligned}
& \left\|f\left(t, y, y^{\prime}\right)\right\| \leq \sqrt[3]{t+\|y\|+\left\|y^{\prime}\right\|}+\frac{1}{41}\left\|y^{\prime}\right\|+3 \ln \left(1+\|y\|^{2}\right), \\
& \left\|I_{1}(y)\right\|=\frac{1}{2}, \quad\left\|\bar{I}_{1}\left(t, y, y^{\prime}\right)\right\|=\frac{1}{2}, \quad \forall t \in J, y, y^{\prime} \in R^{n},
\end{aligned}
$$

so we get $\beta \leq \frac{1}{41}, \beta_{1}=0, \bar{\beta}_{1}=0, \rho_{2}=2, \rho_{3}=\frac{2}{3}, \gamma=2, \phi_{m^{*}}\left(\frac{\gamma \beta}{4}\right) \leq \sqrt{\frac{1}{82}}$.

Hence, $\eta_{1} \leq \sqrt{\frac{8}{41}} \leq 1, \eta_{2} \leq \sqrt{\frac{8}{369}} \leq 1, \eta \leq \sqrt{\frac{8}{41}} \leq 1$. From Theorem 3.1, we get the result. This completes the proof.

\section{Competing interests}

The authors declare that they have no competing interests.

\section{Authors' contributions}

All authors contributed equally to the writing of this paper. All authors read and approved the final manuscript.

\section{Acknowledgements}

The authors express their sincere thanks to the anonymous reviews for their valuable suggestions and corrections for improving the quality of the paper. This work was supported by NNSF of China No. 11431008 and NNSF of China No. 11271261 .

\section{Received: 10 December 2015 Accepted: 29 March 2016 Published online: 14 April 2016}

\section{References}

1. Sun, JP, Wang, XQ: Monotone positive solution of nonlinear beam equations with nonlinear boundary conditions. Math. Probl. Eng. 2011, Article ID 609189 (2011)

2. Yao, QL: Positive solutions of nonlinear beam equations with time and space singularities. J. Math. Anal. Appl. 374, 681-692 (2011)

3. O'Regan, D: Solvability of some fourth (and higher)order singular boundary value problems. J. Math. Anal. Appl. 161, 78-116 (1991)

4. Yang, B: Positive solutions for the beam equations under certain boundary conditions. Electron. J. Differ. Equ. 2005, 78 (2005)

5. Zhang, XG: Existence and iteration of monotone positive solutions for an elastic beam equation with a comer. Nonlinear Anal., Real World Appl. 10, 2097-2103 (2009)

6. Gupta, GP: Existence and uniqueness theorems for the bending of an elastic beam equation. Appl. Anal. 26, 289-304 (1988)

7. Agarwal, RP: On fourth-order boundary value problems arising in beam analysis. Differ. Integral Equ. 2, 91-110 (1989)

8. Bonanno, G, Bella, BD: A boundary value problem for fourth-order elastic beam equations. J. Math. Anal. Appl. 343, 1166-1176 (2008)

9. Han, GD, Xu, ZB: Multiple solutions of some nonlinear fourth-order beam equations. Nonlinear Anal. TMA 68, 3646-3656 (2008)

10. Zhang, XG, Liu, LS: Positive solutions of fourth-order four-point boundary value problems with $p$-Laplacian operator. J. Math. Anal. Appl. 336, 1414-1423 (2007)

11. Zhou, YL, Zhang, XM: New existence theory of positive solutions to fourth order $p$-Laplacian elasticity problems. Bound. Value Probl. 2015, 205 (2015)

12. Feng, MQ: Multiple positive solutions for fourth-order impulsive differential equations with integral boundary conditions and one-dimensional p-Laplacian. Bound. Value Probl. 2011, Article ID 654871 (2011)

13. Afrouzi, GA, Hadjian, A, Radulescu, VD: Variational approach to fourth-order impulsive differential equations with two control parameters. Results Math. 65, 371-384 (2014)

14. Cabada, A, Tersian, S: Existence and multiplicity of solutions to boundary value problems for fourth-order impulsive differential equations. Bound. Value Probl. 2014, 105 (2014)

15. Sun, JT, Chen, HB, Yang, L: Variational methods to fourth-order impulsive differential equations. J. Appl. Math. Comput. 35, 323-340 (2011)

16. Xie, JL, Luo, ZG: Solutions to a boundary value problem of a fourth-order impulsive differential equations. Bound. Value Probl. 2013, 154 (2013)

17. Zhang, XM, Feng, MQ: Positive solutions for classes of multi-parameter fourth-order impulsive differential equations with one-dimensional singular p-Laplacian. Bound. Value Probl. 2014, 112 (2014)

18. Feng, MQ, Qiu, JL: Multi-parameter fourth order impulsive integral boundary value problems with one-dimensional m-Laplacian and deviating arguments. J. Inequal. Appl. 2015, 64 (2015)

19. Guo, DJ, Sun, JX, Liu, ZL: Functional Analysis Method of Nonlinear Ordinary Differential Equations. Shandong Science and Technology Press, Jinan (2005). ISBN:7-5331-1497-3 